\title{
The Micro-Groove Detector
}

\author{
R. Bellazzini ${ }^{\mathrm{a}, \mathrm{b}} \mathrm{M} \mathrm{Bozzo}^{\mathrm{c}}$ A. Brez ${ }^{\mathrm{a}}$ G. Gariano ${ }^{\mathrm{a}}$ L. Latronico $^{\text {a }}$ \\ N. Lumb ${ }^{\text {a }}$ A. Papanestis ${ }^{\text {a }}$ G. Spandre ${ }^{\text {a }}$ M.M. Massai ${ }^{a}$ R. Raffo ${ }^{a}$ \\ M.A. Spezziga ${ }^{a}$ \\ ${ }^{\mathrm{a}}$ INFN-Pisa and University of Pisa, Pisa, Italy \\ ${ }^{\mathrm{b}}$ CERN, CH-1211 Geneva 23, Switzerland \\ ${ }^{\mathrm{c}}$ INFN-Genova and University of Genova, Italy
}

\begin{abstract}
We introduce the Micro-Groove Detector (MGD), a new type of two dimensional position-sensitive gas proportional counter produced using advanced printed circuit board (PCB) technology. The MGD is based on a thin kapton foil, clad with gold-plated copper on both sides. An array of micro-strips at a typical pitch of $200 \mu \mathrm{m}$ is defined on the top metal layer. Using as a protection mask the metal left after the patterning, charge amplifying micro-grooves are etched into the kapton layer. These end on a second micro-strip pattern defined on the bottom metal plane. The two arrays of micro-strips can have an arbitrary relative orientation and so can be used for read-out to obtain 2-D positional information. First results from our systematic assessment of this device are reported: gas gain $>15000$, rate capability above $10^{6} \mathrm{~mm}^{-2} \mathrm{~s}^{-1}$, energy resolution $22 \%$ at $5.4 \mathrm{keV}$, no significant charging or aging effects up to $5 \mathrm{mC} / \mathrm{cm}$, full primary charge collection efficiency even at high drift fields.
\end{abstract}

\section{Introduction}

The introduction of the Micro-Strip Gas Counter (MSGC) [1-3] has spawned the proliferation of a wide variety of gas proportional detectors in which micro-patterned amplifying structures are defined on a substrate using thin-film or micro-electronics technology. In addition to the MSGC, examples of such devices are Micro-Gap Chambers (MGCs) [4], Small Gap Chambers (SGCs) [5,6] and Microdots [7,8]. The main impetus for this development comes from the unprecedented requirements of the tracking systems for the next generation of experiments at the planned high-luminosity colliders such as the Large Hadron Collider (LHC) at CERN. The recent introduction of the Gas Electron Multiplier (GEM) [9] and the Compteur a Trou (CAT) [10] has shown that it is possible to increase the feature size of the smallest electrode structure by almost a factor 10, with apparently no major degradation in performance. This increase in scale allows the use of advanced printed 
circuit board (PCB) technology for detector production as an alternative to the more demanding techniques previously employed. There are several advantages of this new approach, including greater robustness and size of the detector, reduced defect density and lower cost. The main disadvantage is the somewhat reduced speed of the device.

We have built and tested a detector based on a kapton foil, copper-coated on both surfaces. The amplification structures are micro-grooves in the kapton layer, coupled to two micro-strip patterns defined in the first and second metal layers. The two arrays of micro-strips are used for read-out to obtain two dimensional positional information. First results from our systematic assessment of this device, which we call the Micro-Groove Detector (MGD), are reported here. We envisage many possible variations on the present design, including amplifying micro-wells instead of grooves, with either 1-D or 2-D strip read-out [11].

\section{The Micro-Groove Detector concept}

We have adapted advanced PCB technology to produce a new class of detectors of which the MGD is a first example. Our starting-point is a thin kapton foil clad on both sides with a layer of gold-plated copper. The bottom layer is first patterned as a set of closely-spaced strips or pads, then this side of the foil is glued to a thin vetronite or carbon fibre board using the prepregging technique [12]. Because the glued side is now fully insensitive to the etching chemicals, the second metal and kapton layers may be patterned as desired. In the MGD for example, arrays of parallel micro-strips are etched in the top metal layer. The remaining metal is then used as a protection mask for wet etching of the kapton. As a result, the two layers are perforated by a regular pattern of closely-spaced charge amplifying micro-grooves ending on the micro-strip pattern in the lower metal plane, see Fig. 1. Application of a potential difference across the electrodes creates a concentrated electric field in the grooves (Fig. 2) which is high enough to produce sizeable gains when the MGD is enclosed in a suitable gas environment. The gas envelope is formed simply by adding a drift window, separated from the vetronite or carbon-fibre support board by a spacer. The resulting detector is extremely resistant to mechanical shock, overcoming the problems of fragility associated with chambers in which the electrodes are deposited on a thin glass substrate. The electron avalanche charge is collected by the strip pattern on the bottom layer, which provides 1-D positional information. The positive ions flow back towards the top metal electrodes, inducing on them a current signal of equal size but opposite polarity. Reading out these signals provides a second, independent coordinate measurement.

The MGD can be considered as an adaptation of the Micro-Gap Chamber (MGC) concept [4] to PCB technology. The main differences are the increase of the intermetal dielectric thickness from 10 to 50 microns and the reverse geometry of the 


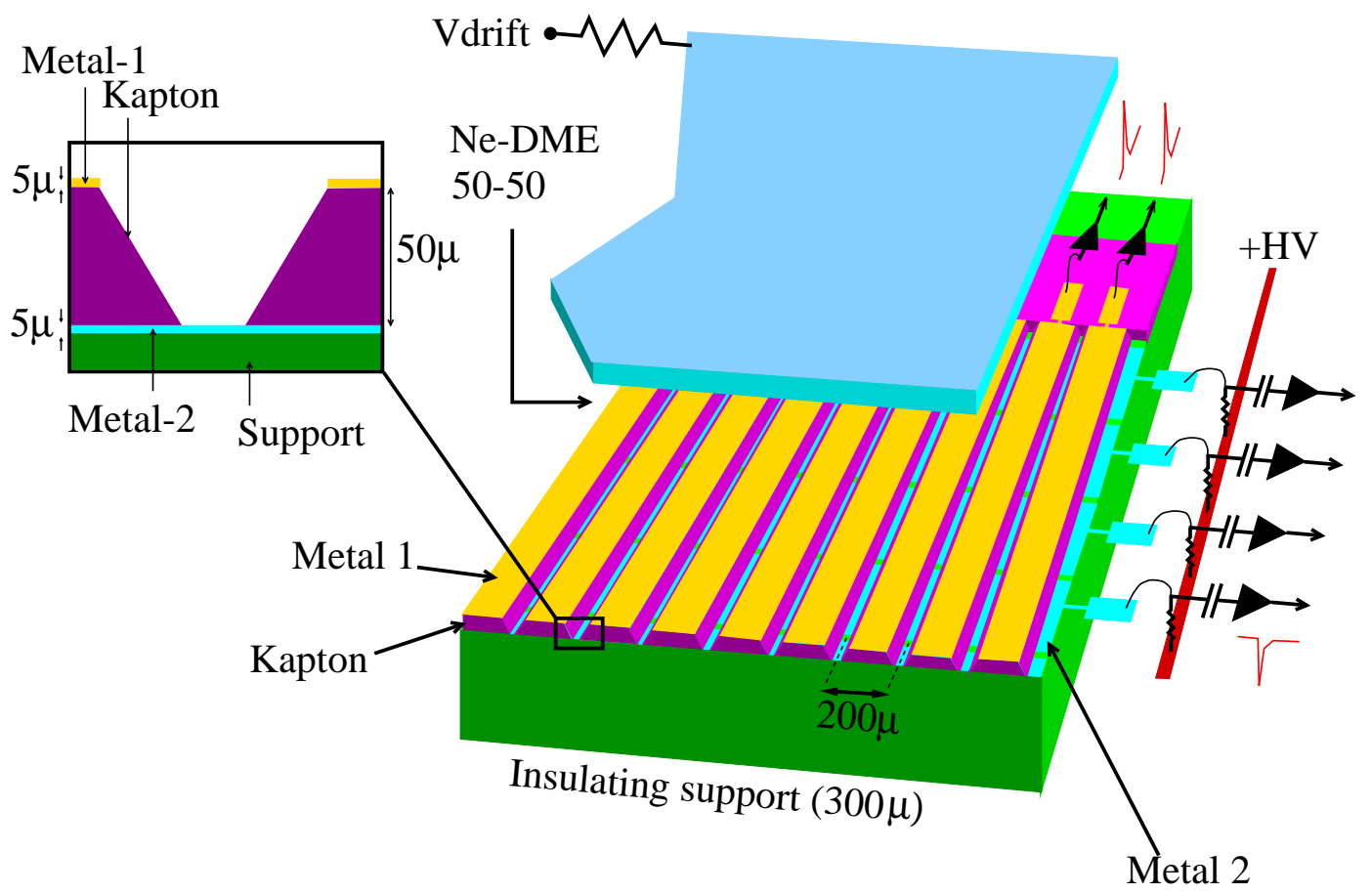

Fig. 1. Schematic diagram of a Micro-Groove Detector with x,y read-out at 90 degrees.

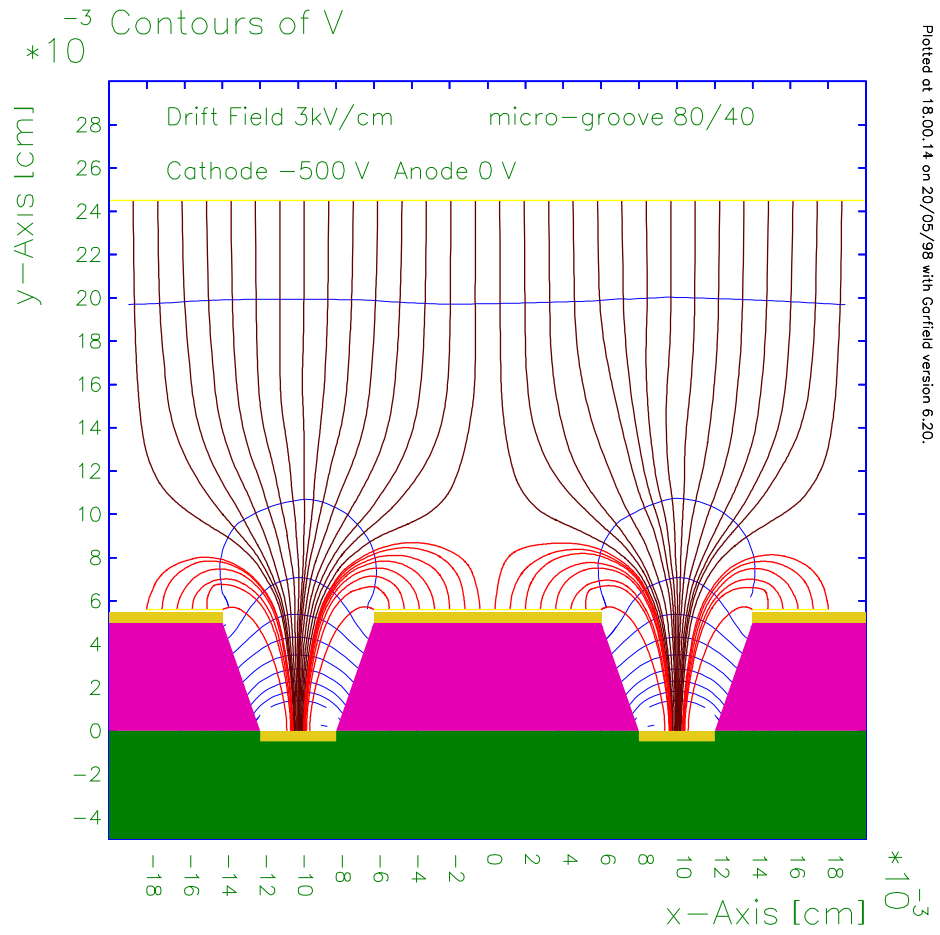

Fig. 2. Equipotential and drift lines (with zero diffusion) for the Micro-Groove Detector. 


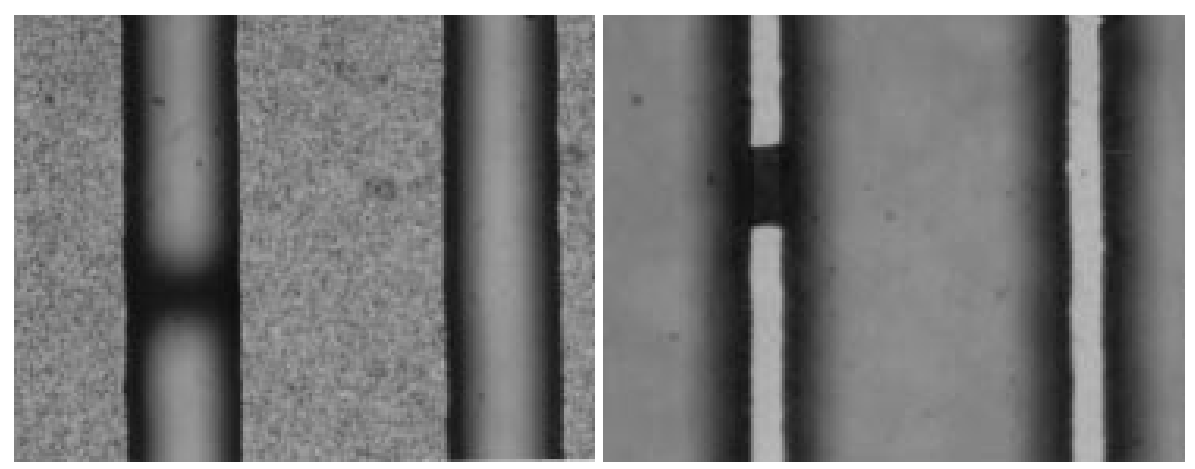

Fig. 3. Microscope photographs of a Micro-Groove Detector, with the focus at the top (left image) and bottom (right image) of the grooves.

amplifying electrodes. In the MGD the cathode is on the top layer and the anode is on the bottom layer, while the arrangement is vice-versa in the MGC. Both modifications contribute to provide an intense electric field all along the $50 \mu \mathrm{m}$ electron amplification path, leading to greater gas gain. Furthermore, the anode width is expanded from 7 to 35 microns and its thickness is 5 microns instead of 0.5 . The result is an increase of the electrode cross-section by a factor 50, which clearly gives much more electrical robustness to the MGD concept.

The use of linear grooves as amplification structures provides full charge collection efficiency even at high drift fields (of the order $6 \mathrm{kV} / \mathrm{cm}$ ). The MGD detector thus retains many of the desirable features of the MSGC/MGC designs (single gain stage, single gas gap, high granularity, mechanical and electrical simplicity, full collection efficiency) and may even be an improvement on the MSGC in a number of areas, such as 2-D sensitivity, lower cost, larger size and greater robustness.

\section{Details of test detector}

The device used for the tests reported in this paper was based upon a $2.6 \times 2.6 \mathrm{~cm}$ square of gold-plated copper-clad kapton. An array of parallel linear strips and grooves was etched in the metal-1 and kapton layers (thicknesses 5 and 50 microns respectively), the distance between groove centers being $200 \mu \mathrm{m}$. The groove width was $70 \mu \mathrm{m}$ in the plane of the metal and approximately $30 \mu \mathrm{m}$ at the bottom of the kapton (the trapezoidal shape of the grooves is a consequence of the etching process). Fig. 3 shows microscope photographs taken from above the grooves, with the focus at the top (left image) and bottom (right image) of the grooves. In the metal-2 layer, an array of charge collection pads, length $4 \mathrm{~mm}$, width $35 \mu \mathrm{m}$ were etched with the long side parallel to the grooves. The gap between two of these pads is clearly visible in Fig. 3. Lines of width $35 \mu \mathrm{m}$, also in the metal-2 layer, were used to interconnect the collection pads to form a $200 \mu \mathrm{m}$ pitch strip array at an angle of 100 milliradians to the top strips. Both sets of read-out strips were terminated in 


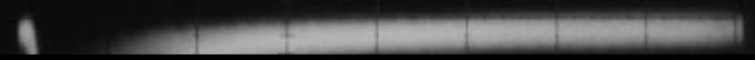

